\title{
Dérives thermiques du capteur de pression capacitif microélectronique
}

\author{
A. Ettouhami $\left({ }^{1}\right)$, A. Essaid $\left({ }^{1, *}\right)$, N. Ouakrim $\left({ }^{1}\right)$, L. Michel $\left({ }^{2}\right)$ \\ et M. Limouri $\left({ }^{1}\right)$ \\ $\left({ }^{1}\right)$ Laboratoire Conception et Systèmes, département de physique, Faculté des Sciences, \\ Rabat, Maroc \\ $\left({ }^{2}\right)$ Département de Génie Mécanique, École Nationale Supérieure d'Ingénieurs de Constructions \\ Aéronautiques, Toulouse, France
}

(Reçu le 25 juin 1996, révisé le 26 novembre 1996 et le 12 mars 1997, accepté le 28 mars 1997)

\section{PACS.07.Cm - Micromechanical devices and systems}

Résumé. - Les dérives thermiques du capteur de pression capacitif microélectronique sont analysées par la méthode des éléments finis. Différentes conditions aux limites représentant une large gamme de support de capteur ont été envisagées : capteur à base libre, capteur à base fixe et capteur collé à un support d'alumine. Dans chaque cas, la réponse thermique du capteur a été déterminée en fonction des dimensions du capteur afin de repousser la température de flambage et réduire en conséquence la sensibilité thermique. Pour certains capteurs très sensibles à la pression, cette température est très faible et les dérives thermiques sont importantes. Ainsi un capteur à base fixe (membrane de rayon $1000 \mu \mathrm{m}$, et épaisseur $12 \mu \mathrm{m}$, distance entre armatures $3,5 \mu \mathrm{m}$ ) présente une sensibilité thermique de $-200 \mathrm{~Pa}^{\circ} \mathrm{C}^{-1}$ au-dessus de $80^{\circ} \mathrm{C}$. Un capteur collé à un support d'alumine, de membrane plus large (rayon $1800 \mu \mathrm{m}$ ) possède une ${ }_{\diamond}$ sensibilité $^{\circ}$ thermique de $3,1 \mathrm{~Pa}^{\circ} \mathrm{C}^{-1}$ au-dessous de $-100^{\circ} \mathrm{C}$.

\begin{abstract}
The thermal drifts of microelectronic capacitive pressure sensor have been analysed by finite-element method. Various boundary conditions representing a wide gamut of support of sensor have been considered: sensor with free base, sensor with fixed base and sensor attached to a support of alumina. For every case, the thermal response of sensor have been determined as a function of sensor dımensions in order to push far the buckling temperature and hence reduce the thermal sensitivity. For some sensors very sensitive to pressure, this temperature is small and the thermal drifts are important. A sensor with fixed base having a diaphragm of $1000 \mu \mathrm{m}$ of radius and $12 \mu \mathrm{m}$ of thickness and having a $3.5 \mu \mathrm{m}$ of plate separation presents a thermal sensitivity of $-200 \mathrm{~Pa}{ }^{\circ} \mathrm{C}^{-1}$ above $80^{\circ} \mathrm{C}$. A sensor attached to a support of alumina, having a more wide diaphragm (1800 $\mu \mathrm{m}$ of radius) presents a thermal sensitivity of $3.1 \mathrm{~Pa}^{\circ} \mathrm{C}^{-1}$ below $-100^{\circ} \mathrm{C}$.
\end{abstract}

\section{Introduction}

$\mathrm{Au}$ cours de ces dernières années, l'automatisation, le contrôle et la surveillance de la plupart des processus ont induit un besoin croissant en capteurs. En effet, dans la plupart des applications, le manque de capteurs adéquats et d'actionneurs pour coupler l'électronique de

(*) Auteur auquel doit être adressée la correspondance

(C) Les Éditions de Physique 1997 
contrôle avec l'environnement extérieur est le principal problème dans le développement de nouveaux systèmes. Des recherches, mettant à profit les progrès de la microélectronique et d'autres techniques compatibles, ont permis d'une part de réaliser, à faible coût, des capteurs et des actionneurs miniaturisés et de hautes performances, et d'autre part d'élargir le spectre d'utilisation des capteurs.

Le capteur de pression est l'un des capteurs les plus importants. Son champ d'application s'étend à plusieurs domaines : l'automobile, la médecine, les processus industriels etc. Malgré les progrès enregistrés dans cette catégorie de capteur [1-4], certains problèmes restent posés tels que les dérives thermiques qui peuvent masquer entièrement ou partiellement la mesure et particulièrement dans le cas de faibles variations de pression. L'origine de la dérive thermique observée pour les capteurs de pression capacitifs microélectroniques (absolus et différentiels) est la différence des coefficients de dilatation thermique entre le silicium, le pyrex et le boîtier d'encapsulation. Cette différence engendre des forces de traction ou de compression dans la membrane, modifie sa déflexion et par suite la réponse du capteur.

Très peu de travaux ont été effectués sur ce sujet. Par exemple Wise, en 1982 [1], a déterminé la sensibilité thermique du capteur capacitif de pression dans un intervalle réduit de température -30 à $70^{\circ} \mathrm{C}$. Il a déterminé, par la méthode des différences finies, la déflexion de la membrane en fonction de la température, sans tenir compte des autres dimensions du capteur et en supposant que les coefficients de dilatation thermique du silicium et du pyrex sont constants. Lin et al. en 1994 [8] ont étudié la distribution des contraintes thermiques dans un capteur piézorésistif de pression. Notre contribution consiste à étudier le comportement thermique du capteur capacitif dans des plages importantes de température, mettant ainsi en évidence le phénomène de flambage thermique du capteur. Ce phénomène qui se produit à certaines températures, peut conduire à une destruction du capteur. Cette étude est effectuée en considérant l'ensemble de la structure du capteur et en tenant compte de la variation des coefficients de dilatation thermique du silicium et du pyrex en fonction de la température.

\section{Principe et modélisation du capteur}

La figure 1 représente le schéma de princıpe du capteur de pression capacitif. Une pastille de silicium micro-usinée des deux faces constitue l'armature déformable. Une couche métallique, déposée sur un substrat en pyrex 7740 , constitue la deuxième armature du condensateur. L'assemblage des deux électrodes est réalisé par la technique de la soudure thermoélectrique évitant ainsi des effets secondaires dus aux couches intercalaires. Sous l'action d'une pression uniformément répartie, l'armature en silicium se déforme et produit ainsi une variation de la capacité du condensateur. La valeur de la capacité, en réponse à la pression appliquée, est exprimée par :

$$
C=\int_{S} \frac{\varepsilon \mathrm{d} S}{(d-w(x, y))}
$$

où $C$ est la capacité à la pressıon différentielle $P, S$ est la surface de la membrane, $d$ est la distance entre les armatures du capteur au repos, $\varepsilon$ est la permittivité du diélectrique séparant les deux armatures du condensateur, $w(x, y)$ est la déflexion de la membrane au point de coordonnées $x, y$.

La déflexion $w(x, y)$ de la membrane dépend essentiellement de la pression différentielle qui lui est appliquée, de sa forme et de ses dimensions géométriques. Elle est également fonction de la température. Cette dépendance en température est liée principalement à la différence des coefficients de dilatation thermique entre le silicium et les matériaux de couplage (pyrex et support) [1]. 


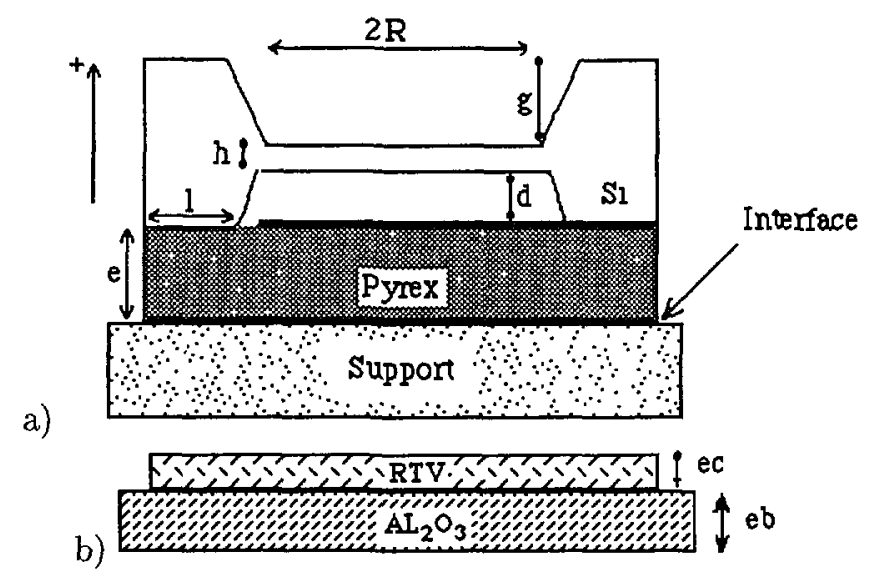

Fug. 1. - Capteur de pressıon capacitıf microélectronique. a) schéma de princıpe, b) exemple de support.

[Microelectronic capacitıve pressure sensor a) Principle scheme, b) example of support ]

Les déflexions et les efforts aux bords de la membrane du silicium dépendent des dimensions du capteur (épaisseur du pyrex, surface de soudure, épaisseur du bord du silicium...) et de la façon dont il est fixé sur le support, ce qui rend difficile la formulation des conditions aux limites. Pour mieux modéliser le comportement thermique du capteur, l'étude doit être effectuée sur l'ensemble de la structure et non pas sur la membrane seule Dans ce cas, la résolution du problème nécessite une étude tridimensionnelle, qu ne peut être effectuée de façon simple par voie analytique, et passe par la réalisation d'un calcul numérique par la méthode des éléments finis. Nous avons utilisé le logiciel de calcul de structures SAMCEF [9] pour modéliser et étudier le comportement thermique du capteur. Le logiciel SAMCEF est un produit commercial qui permet de faire, entre autres, des analyses statiques, dynamiques et thermiques des problèmes élastiques linéaires et non linéaires. Il est exploité dans les industries de constructions mécaniques, aéronautiques, dans le domaine du transport, du géne civil, etc.

Afin de bien maîtriser les dérives thermiques du capteur, les conditions aux limites au niveau de l'interface pyrex-support ont été choisies de manière à représenter d'abord les cas extrêmes de supporf : base libre où chaque point de l'interface se dilate librement et base fixe où tous les points de l'interface ont des déplacements nuls suivant toutes les directions (cas de support rigide). Ensuite, un cas plus réaliste de support a été étudié, pour lequel les conditions aux limites se situent entre les deux cas extrêmes précédentś.

\section{Flambage du capteur}

Lorsque les contraintes d'origine thermique dans une membrane sont compressives et suffisamment grandes pour atteindre une valeur critique, l'équilibre peut devenır instable et un flambage peut avoir lieu en absence de toute force extérıure [5]. Ce phénomène est appelé flambage thermique et la température correspondant à la charge critique est appelée température de flambage. Au-delà de cette charge critique, les déformations ne sont plus proportionnelles aux efforts appliqués (phénomène de non linéarité) Ces déformations peuvent devenır consıdérables au point de provoquer rapidement l'effondrement de la structure. 
Tableau I. - Propriétés mécanıques du silicium et du pyrex 7740

[Mechnical properties of silicon and pyrex materials.]

\begin{tabular}{|l|c|c|c|}
\hline Matériau & $\begin{array}{c}\text { Module d'Young } \\
E\left(10^{9} \mathrm{~Pa}\right)\end{array}$ & $\begin{array}{c}\text { Coefficient de } \\
\text { Poisson } \nu\end{array}$ & $\begin{array}{c}\text { Coefficient de dilatation } \\
\text { thermique } \alpha\left(10^{-6}{ }^{\circ} \mathrm{C}^{-1}\right)\end{array}$ \\
\hline Silicium & 169 & 0,066 & 2,3 \\
\hline Pyrex & 60 & 0,25 & 3,1 \\
\hline Colle RTV & $6,894 \times 10^{-3}$ & $\circ 0,4$ & 800 \\
\hline $\mathrm{AL}_{2} \mathrm{O}_{3}$ & 276 & 0,22 & 7,1 \\
\hline
\end{tabular}

Tableau II. - Configurations étudiées et plages de varıations de leurs dimensions. [Studied configurations and variation domains of their dimensions.]

\begin{tabular}{|c|c|c|c|c|c|}
\hline Type & Configuration & $R(\mu \mathrm{m})$ & $e(\mu \mathrm{m})$ & $e c(\mu \mathrm{m})$ & $e b(\mu \mathrm{m})$ \\
\hline CP1 & base fixe & 1000 & 200 & - & - \\
\hline CP2 & base fixe & 1000 & $100-400$ & - & - \\
CP3 & base libre & $800-1600$ & 200 & - & - \\
CP4 & base collée à l'alumine & $1200-1800$ & 200 & 200 & 400 \\
\hline
\end{tabular}

L'intêret est porté à la détermination des deux premiers modes de flambage thermique du capteur capacitif de pression et des températures critiques correspondantes. Les propriétés mécaniques des matériaux utilisés dans les simulations sont celles mentionnées dans la littérature et sont regroupées dans le tableau $\mathrm{I}$.

Les dimensions utilisées (voir Fig. 1) dans toutes les simulations sont les suivantes : $h=$ $12 \mu \mathrm{m}, d=3,5 \mu \mathrm{m}, g=200 \mu \mathrm{m}$ et $l=300 \mu \mathrm{m}$. Les autres dimensions varient suivant les cas étudiés ; leurs plages de variation sont regroupées dans le tableau II.

La figure 2 montre que le flambage est localisé au niveau de la membrane, le premier mode de flambage est symétrique et le deuxième mode de flambage est antisymétrique. Ceci est valable dans les deux cas extrêmes du support (base fixe et base libre). Notons que le deuxième mode ne peut se présenter que par empêchement de l'apparition du premier mode. Les simulations montrent que le flambage se produit à des températures positives $\left(T>0^{\circ} \mathrm{C}\right)$ pour les capteurs à base fixe et à des températures négatives $\left(T<0^{\circ} \mathrm{C}\right)$ dans le cas des capteurs à base libre. Comme exemple, le capteur à base fixe CP1 présente son premier mode de flambage à une température voisine de $105^{\circ} \mathrm{C}$. Un capteur à base libre ayant une membrane plus fragile, de $1600 \mu \mathrm{m}$ de rayon et de $10 \mu \mathrm{m}$ d'épaisseur présente un flambage au voisinage de $-106^{\circ} \mathrm{C}$. Ceci s'explique comme suit :

- Base libre : du fait que le coefficient de dilatation thermique du pyrex est supérieur à celui du silicium, les dilatations du premier matériau sont plus importantes que celles du second. Ainsi pour des températures positives, les efforts exercés par le pyrex sur le silicium ne sont que des efforts de traction. Inversement lorsqu'il s'agit des températures négatives, le pyrex engendre l'apparition des efforts de compression sur la membrane, conduisant ainsi au flambage du capteur.

- Base fixe : pour des températures positives, la membrane est soumise à des efforts de compression dues à la fixation de l'interface support-pyrex, et subit par conséquent un flambage thermique. 

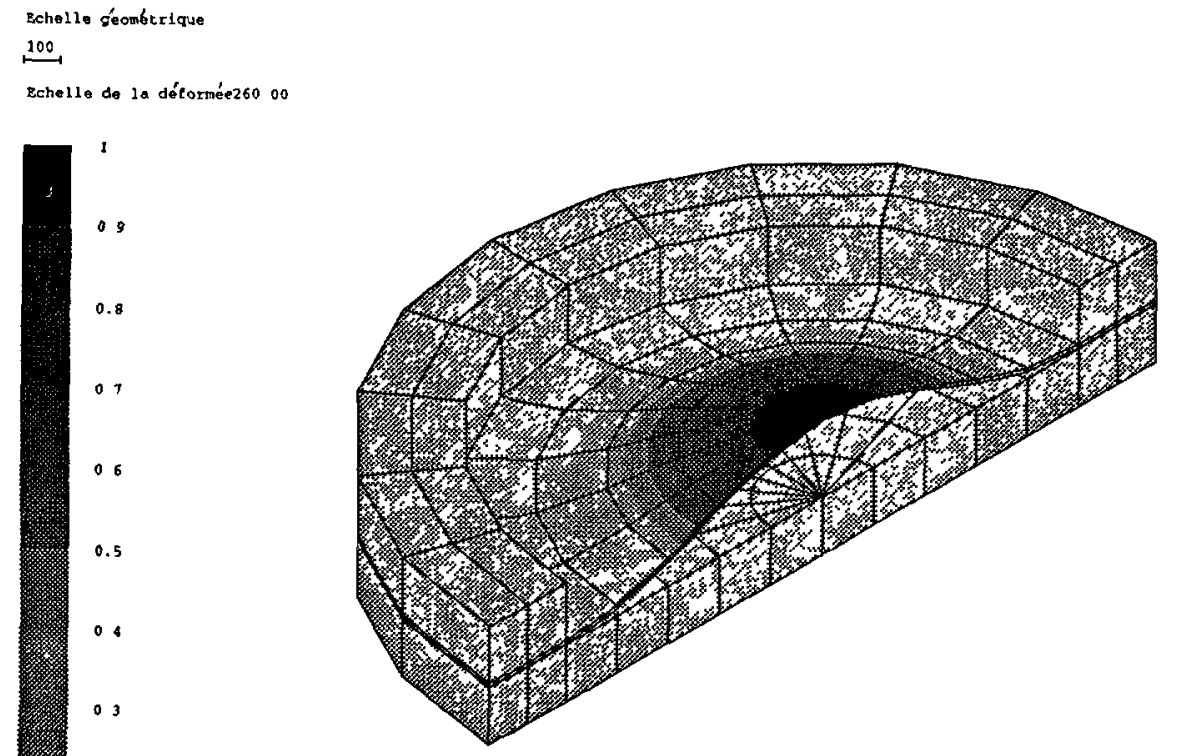

a)

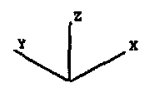

Fig. 2. - Représentation en trois dimensions de la moitié du capteur CP1 en état de flambage. a) : premier mode de flambage, b) : deuxième mode de flambage (déformée réelle $=$ déformée sur la figure/échelle de la déformée).

[3D representation of the half of the sensor CP1 at the buckling state. a) First mode of buckling, b) second mode of buckling.]

\section{Cas limites de dérives thermiques}

La réponse du capteur au voisinage de la température de flambage a été déterminée pour les deux cas extrêmes de conditions aux limites : base fixe et base libre. Cette réponse est étudiée en tenant compte de la variation des coefficients de dilatation thermique en fonction de la température (Fig. 3).

3.1. BASE FIXE. - Les figures 4 et 5 représentent respectivement la déflexion relative au centre de la membrane (différence de déflexion entre le centre de la membrane et le centre du pyrex) et la valeur de la capacité en fonction de la température pour un capteur ayant une base fixe. On remarque d'après la figure 4 qu'entre $0^{\circ} \mathrm{C}$ et un seuil de température $\left(\sim 80^{\circ} \mathrm{C}\right)$ inférieur à la température de flambage $\left(105^{\circ} \mathrm{C}\right)$ la déflexion varie presque linéairement et assez faiblement ; au-delà de ce seuil la variation devient importante et non linéaire. Ceci est en accord avec la définition précédente. Notons que le sens positif choisi est celui indiqué sur la figure 1 . 

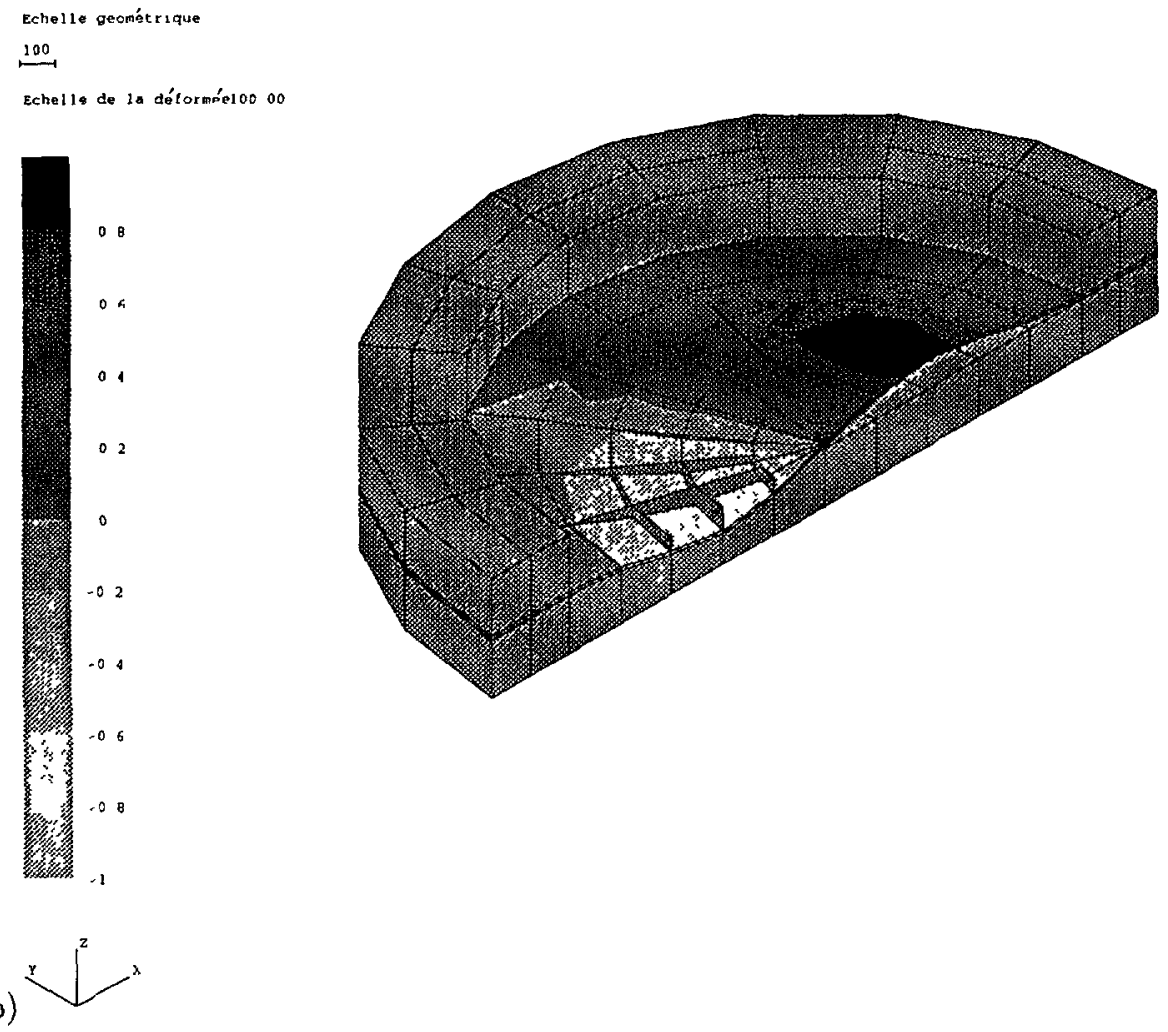

Fig. 2. - (Sunte).

[Continued]

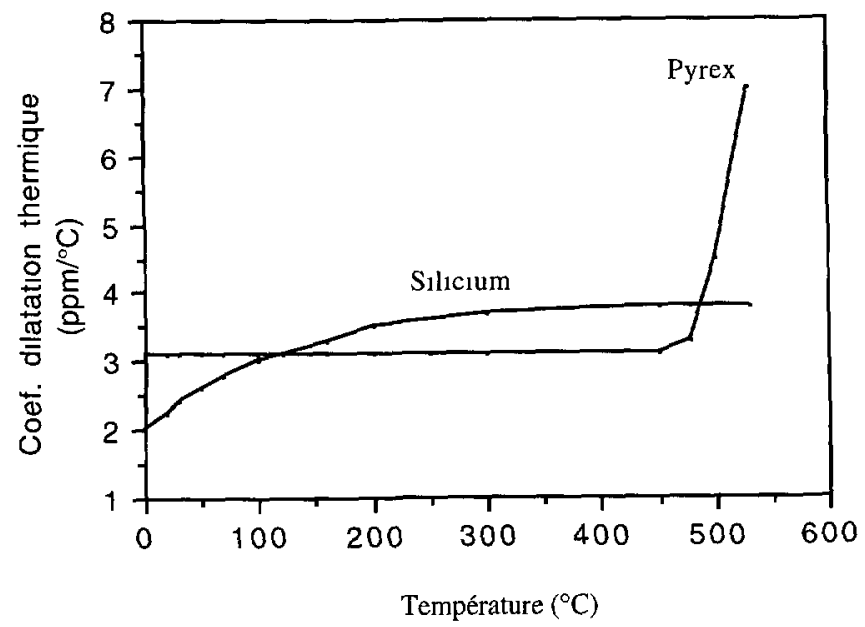

Fig 3. - Coefficıents de dilatation thermıque du silicium et du pyrex en fonction de la température. [Thermal coefficients of expansion as function of the temperature for slicon and pyrex materials.] 


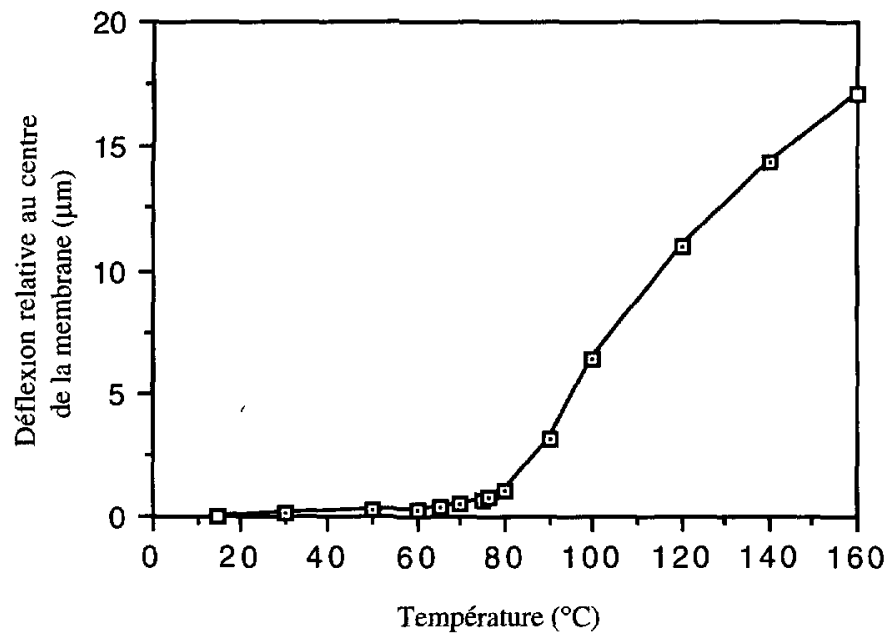

Fig. 4. - Déflexıon relative du centre de la membrane en fonction de la température pour le capteur à base fixe CP1.

[Relative deflection of the diaphragm center as function of temperature for the sensor with fixed base: CP1.]

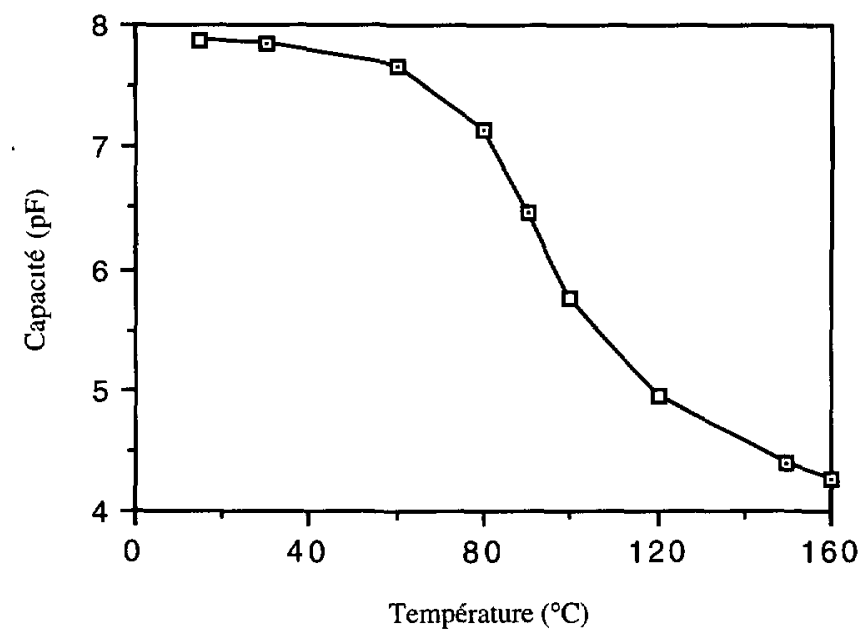

Fig. 5. - Réponse en température du capteur à base fixe CP1.

[Thermal response of the sensor with fixed base: CP1.]

En utilisant la courbe de la figure 5 et le programme de calcul de la capacité en fonction de la pression, développé dans [6] et [7], on déduit que la sensibilité en température est équivalente à $-13 \mathrm{~Pa}^{\circ} \mathrm{C}^{-1}$ dans l'intervalle $(0 \text { à } 80)^{\circ} \mathrm{C}$. Cette valeur est faible et en accord avec les travaux antérieurs [1]. Au-delà de $80^{\circ} \mathrm{C}$, le capteur devient très sensıble à la température, cette sensibilité est de l'ordre de $-200 \mathrm{~Pa}^{\circ} \mathrm{C}^{-1}$. 


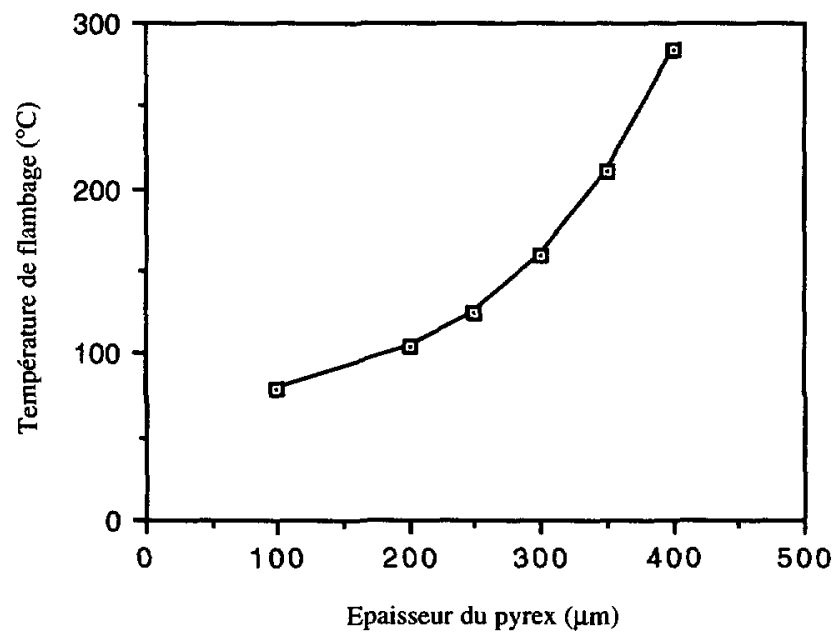

Fig. 6. - Température du premier mode de flambage du capteur à base fixe CP2 en fonction de l'épaisseur du substrat de pyrex.

[Temperature of the fisrt mode of buckling of the sensor with fixed base: CP2, as function of pyrex thickness.]

L'influence de différents paramètres sur la réponse du capteur à base fixe a été étudiée afin de diminuer les dérives thermiques. Nous avons remarqué que le choix de petites surfaces et de grandes épaisseurs de la membrane tend à augmenter la température de flambage et diminuer par conséquent la sensibilité à la température. Cependant ce choix fait diminuer également la sensibilité à la pression. Nous avons constaté également que le choix de grandes épaisseurs de substrat de pyrex tend à augmenter la température de flambage (Fig. 6) sans modifier la sensibilité à la pression.

3.2. BASE LIBRE. - Comme nous l'avons signalé précédemment, le capteur à base libre ne présente pas de flambage à des températures positives. La membrane est soumise à des efforts de traction seulement. La courbe de la figure 7 donne un exemple de réponse du capteur à base libre pour différentes valeurs du rayon de la membrane. La variation relative de la déflexion au centre de la membrane s'explique comme suit :

- Pour des températures inférieures à $120^{\circ} \mathrm{C}$ où le coefficient de dilatation thermique du pyrex est supérieur à celui du silicium, chaque élévation de température entraine une variation de la déflexion dans le même sens que la précédente. La superposition de ces déplacements explique l'allure croissante de la courbe dans l'intervalle $(0 \text { à } 120)^{\circ} \mathrm{C}$.

- Pour des températures supérieures à $120^{\circ} \mathrm{C}$, le coefficient de dilatation thermique du pyrex est inférieur à celui du silicium. Les déflexions produites par des élévations de température ont un sens opposé à celui de l'intervalle $(0 \text { à } 120)^{\circ} \mathrm{C}$ et par conséquent la courbe décroît jusqu'à une température de $270^{\circ} \mathrm{C}$ environ où les dilatations thermiques des deux matériaux sont égales. À cette température, aucune force n'est appliquée sur les deux matériaux et la déflexıon relative est donc nulle. Au-delà de $270^{\circ} \mathrm{C}$ le phénomène 


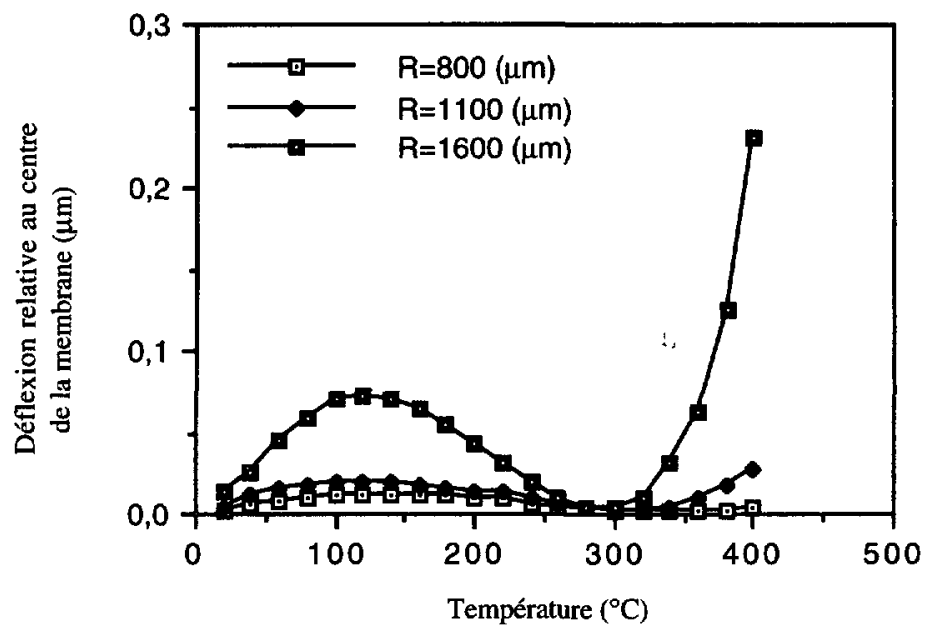

Fig. 7. - Déflexion relative du centre de la membrane en fonction de la température pour le capteur à base libre CP3.

[Relative deflection of the diaphragm as a function of temperature for the sensor with free base: CP3.]

est inversé ; des forces de compression apparaissent sur la face inférieure de l'armature en silicium. Ces forces expliquent la forte croissance de la courbe dans le sens positif et peuvent produire un flambage à des températures très élevées.

La déflexion relative entre le silicium et le substrat de pyrex reste faible par rapport à celle du capteur à base fixe dans l'intervalle $(0 \text { à } 400)^{\circ} \mathrm{C}$.

\section{Réponse thermique du capteur collé sur son support}

Une des techniques courantes d'encapsulation des capteurs de pression sur silicium est d'attacher le capteur à un substrat d'alumine $\left(\mathrm{Al}_{2} \mathrm{O}_{3}\right)$ en utilisant une colle adhésive de type RTV [8] (Fig. 1b). Ayant un faible module d'élasticité, cette colle permet d'atténuer les contraintes engendrées par l'alumine, et de diminuer leur influence sur la membrane de silicium.

La déflexion relative au centre de la membrane en fonction de la température pour différentes valeurs du rayon de la membrane du capteur CP4 est représentée sur la figure 8 . On remarque que le capteur présente un flambage à des températures négatives. En effet, les coefficients de dilatation thermique des autres matériaux (pyrex, RTV, alumine) sont tous supérieurs à celui du silicium dans la gamme de température étudiée, et les forces engendrées par ces matériaux sur le silicium sont compressives lorsque les températures deviennent négatives.

La figure 8 et la figure 9 montrent que la membrane de silicium et le substrat de pyrex (armatures du capteur) se rapprochent pour des températures positives, et s'éloignent pour des températures négatives. La valeur de la capacité en fonction de la température pour un capteur CP4 ayant une membrane circulaire de $1800 \mu \mathrm{m}$ est représentée sur la figure 10 . Un capteur ayant ces dimensions, destiné à fonctionner dans une gamme de pression de $(0-530) \mathrm{Pa}$, possède une sensibilité en température de $0,32 \mathrm{~Pa}^{\circ} \mathrm{C}^{-1}$ pour des températures supérieures à $-60{ }^{\circ} \mathrm{C}$ et de $3,1 \mathrm{~Pa}^{\circ} \mathrm{C}^{-1}$ au-dessous de $-100{ }^{\circ} \mathrm{C}$. 


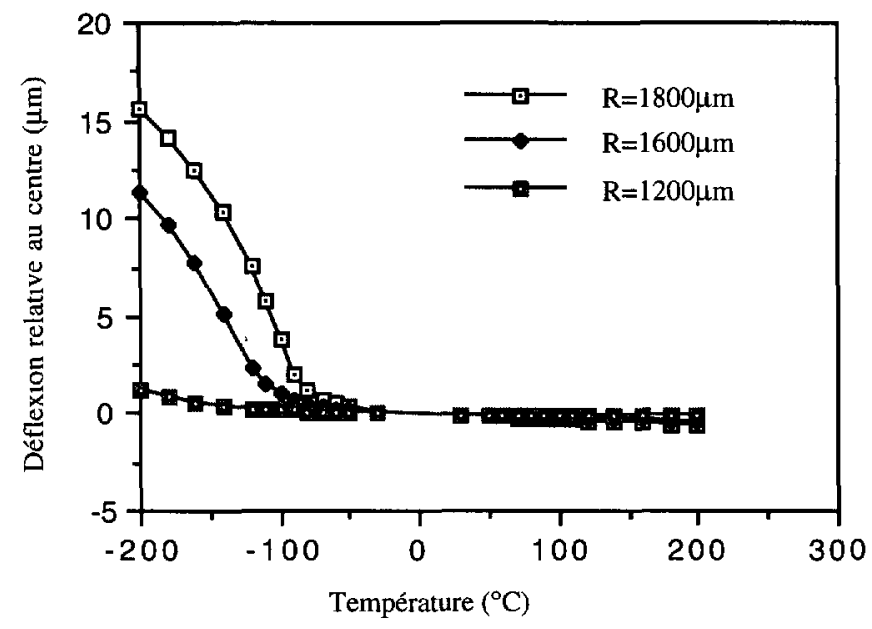

Fig. 8. - Déflexion relative du centre de la membrane en fonction de la température pour le capteur collé à un support d'alumine : $\mathrm{CP} 4$.

[Relative deflection of the diaphragm center as function of temperature for the sensor attached to alumina. CP4 ]

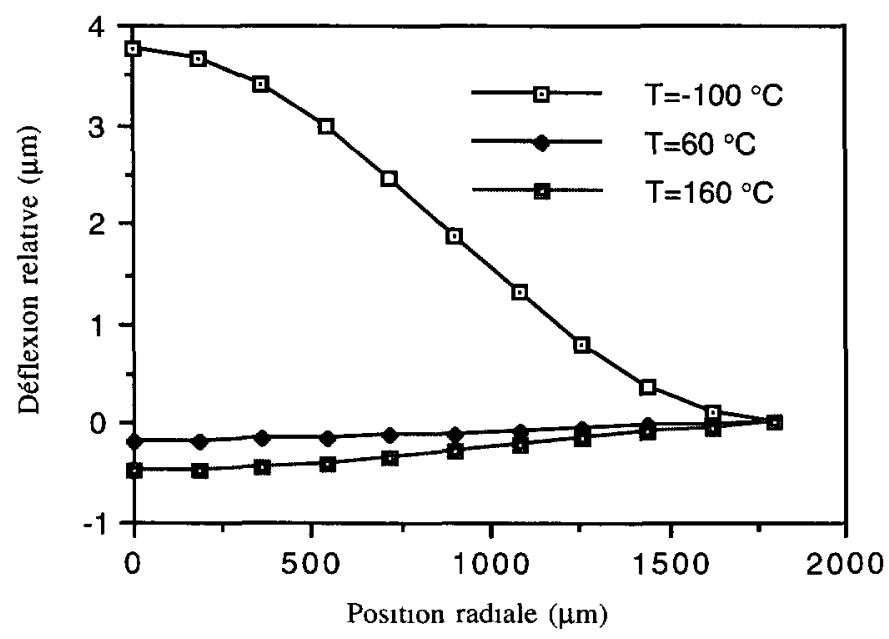

Fig. 9 - Déflexion relative de la membrane à différentes températures pour le capteur collé à l'alumine : CP4.

[Relative deflection of the diaphragm of the sensor attached to alumina: CP4, for various temperatures.]

Notons que l'épaisseur de la colle et celle du boitier n'ont pas d'influence notable sur la réponse du capteur, alors qu'une augmentation de l'épaisseur du pyrex fait diminuer légèrement la sensibilité à la température. 


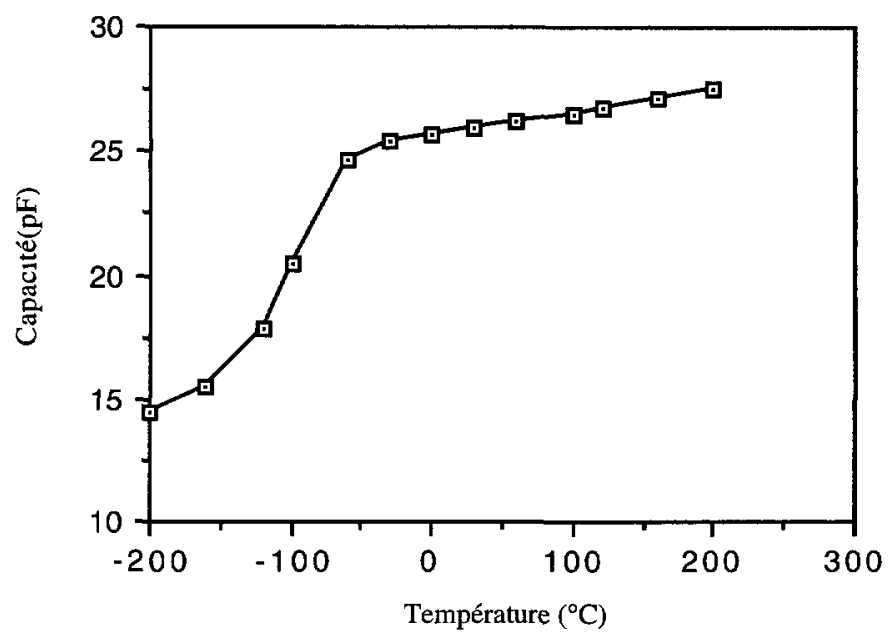

Fig. 10. - Réponse thermique du capteur collé à l'alumıne : CP4 $(R=1800 \mu \mathrm{m})$.

[Thermal response of the sensor attached to alumina: CP4 $(R=1800 \mu \mathrm{m})]$

\section{Conclusion}

Les dérives thermiques du capteur de pression capacitif microélectronique sont dues essentiellement à la différence de coefficients de dilatation thermique du silicium et des matériaux de couplage (pyrex et boitier). Ces dérives sont analysées par la méthode des éléments finis. Elles peuvent être importantes pour certaines dimensions et sont liées au phénomène de flambage thermique de l'armature déformable du capteur. Les conditions de fixation du capteur sur le support jouent un rôle particulièrement important dans ce phénomène. Le capteur à base fixe présente un flambage thermique à des températures positives. Le capteur à base libre présente un flambage à des températures négatives. Le capteur collé sur un support d'alumine à l'aide d'une colle RTV présente une sensibilité thermique de $6 \times 10^{-3} \mathrm{EM}^{\circ} \mathrm{C}^{-1}$ au-dessous de $-100^{\circ} \mathrm{C}$ qui représente la température de flambage du capteur ayant les dimensions citées plus haut (EM : étendue de mesure en pression).

\section{Bibliographie}

[1] Lee K W. and Wise K.D., SENSIM. A Simulation Program for Solid-State Pressure Sensors, IEEE Trans. on Electron Devıces 29 (1982) 34-41.

[2] Ko W H., Bao M.H. and Hong Y.D., A High-Sensitivity Integrated-Circuit Capacitive Pressure Transducer, IEEE Trans. on Electron Devices 29 (1982) 48-56.

[3] Ji J., Cho S.T., Zhang Y., Najafi K. and Wise K.D., An Ultraminiature CMOS Pressure Sensor for a Multiplexed Cardiovascular Catheter, IEEE Trans. on Electron Devices 39 (1992) 2260-2267.

[4] Rosengren L., Soderkvist J. and Smith L., Micromachined sensor structures with linear capacitive response, Sensors and Actuators A 31 (1992) 200-205. 
[5] Parkus Heinz, Thermoelasticity, Springer-Verlag, Wien New York second edition (1976).

[6] Essaid A., Ettouhami A., Kerouad M. and Limouri M., Contribution to study of capacitive pressure sensors, Eurosensors, Third conference on Sensors and their applications (Cambridge 22-24 September 1987, England) Proceeding of programme and abstracts, pp. 186-187.

[7] Ettouhami A., Modélisation, intégrabilité et limites de fonctionnement des capteurs de pression capacitifs, Thèse de $3^{\mathbf{e}}$ cycle, Faculté des Sciences (Rabat, Maroc, 1987).

[8] Lin Y.C., Hesketh P.J. and Schuster J.P., Finite-element analysis of thermal stresses in a silicon pressure sensor for various die-mount materials, Sensors and Actuators $A \mathbf{4 4}$ (1994) 145-149.

[9] SAMCEF, version 5.1, M1, description générale de samcef Documentation Samtech (Liège, 1994). 